\title{
Morbilidad de la linfadenectomía lumboaórtica laparoscópica en pacientes con tumor testicular no seminomatoso en estadío clínico A*
}

\author{
Drs. OCTAVIO A. CASTILLO C. ${ }^{1,2,3}$, JORGE RIOJA Z. ${ }^{1}$, IVAR VIDAL M. ${ }^{1}$, \\ MIGUEL FERIA F. ${ }^{1}$, RAFAEL SÁNCHEZ S. ${ }^{1}$ \\ Unidad de Urología Clínica Indisa. \\ 2 Facultad de Medicina, Universidad Andrés Bello. \\ 3 Facultad de Medicina, Universidad de Chile. \\ Santiago, Chile.
}

\begin{abstract}
Operative morbidity in laparoscopic retroperitoneal lymph node disection in clinical stage A non-seminomatous testis cancer
\end{abstract}

Background: Retroperitoneal Lymph node dissection is part of the treatment of patients with nonseminomatous germ cell tumors stage I (NSGCT). Aim: To report the immediate surgical outcomes of patients subjected to laparoscopic retroperitoneal lymph node dissection. Material and Methods: Prospective analysis of the surgical outcomes of 118 patients aged 15 to 44 years, who underwent Laparoscopic Retroperitoneal Lymph Node Dissection (L-RPLND) for NSGCT stage I. Results: Median blood loss was $50 \mathrm{cc}$ (range 10-1.000 cc), median operative time was $120 \mathrm{~min}$. (range 60-300 min). Mean hospital stay was 41.4 hours. Twelve patients $(10.2 \%)$ had operative complications. Ten had vascular injuries $(8.5 \%)$, one had a duodenal injury $(0.85 \%)$ and one had an acute pulmonary edema $(0.85 \%)$. Eight lesions were repaired laparoscopically and three required conversion to open surgery. There was no mortality in this series. Conclusions: L-RPLND had an acceptable complication rate in this series of patients. Most of the complications could be resolved laparoscopically.

Key words: Lymph node dissection, laparoscopy, complications, testicular cancer.

\section{Resumen}

Introducción: La linfadenectomía retroperitoneal lumboaórtica es el método de elección en el tratamiento de los pacientes con cáncer testicular no seminoma en estadío A. Este trabajo muestra los resultados inmediatos de una serie de 118 pacientes operados por vía laparoscópica. Material y Método: Se analizan los resultados quirúrgicos de 118 pacientes portadores de un cáncer testicular no seminoma estadío clínico A. Resultados: La media de edad fue de 29 años. La mediana de sangrado fue de $50 \mathrm{cc}$, el tiempo quirúrgico tuvo una mediana de 120 minutos y el tiempo de hospitalización tuvo una media de 41,4 horas. Complicaciones intraoperatorias ocurrieron en 12 pacientes (10,2\%): 10 lesiones hemorrágicas $(8,5 \%)$, 1 lesión duodenal $(0,85 \%)$ y 1 edema pulmonar agudo $(0,85 \%)$. De las 11 lesiones quirúrgicas, 8 fueron

* Recibido el 28 de Julio de 2009 y aceptado para publicación el 25 de Agosto de 2009.

Correspondencia: Dr. Octavio A. Castillo

Apoquindo 3990, Of. 809, Santiago, Chile. Código postal: 7550112. Fax: (56-2) 2282524

E-mail: octaviocastillo@vtr.net 
reparadas por vía laparoscópica, requiriendo conversión a cirugía abierta 3 casos. No hubo mortalidad en la serie. Conclusiones: La linfadenectomía lumboaórtica laparoscópica es un procedimiento estandarizado, con una tasa de complicaciones aceptable, la mayoría de las cuales puede ser resuelta en forma laparoscópica.

Palabras clave: Linfadenectomía lumbo-aórtica, laparoscopía, complicaciones, cáncer de testículo.

\section{Introducción}

Actualmente la Linfadenectomía Retroperitoneal Lumboaórtica (LRLA) es considerada el estándar dorado para la etapificación y tratamiento de los tumores de células germinales no seminoma etapa A. Si bien es cierto, con la disponibilidad de un esquema de quimioterapia altamente efectivo y el uso de nuevas técnicas de imagen para la etapificación de estos pacientes, las indicaciones para la LRLA son debatibles, este procedimiento entrega un manejo integral exitoso en este grupo de pacientes $^{1}$.

Por otro lado, la morbilidad y el carácter invasivo de este procedimiento son aspectos relevantes que se deben tomar en cuenta. En este sentido, la vía de abordaje laparoscópica nos permite realizar un procedimiento menos invasivo $\mathrm{y}$, en manos experimentadas, con un porcentaje aceptable de complicaciones. El objetivo de este trabajo es reportar los resultados inmediatos de una serie consecutiva de 118 pacientes sometidos a una Linfadenectomía Retroperitoneal Lumboaórtica Laparoscópica (LRLA-L).

\section{Material y Método}

Entre junio de 1993 y mayo de 2007, 118 pacientes portadores de un tumor de células germinales no seminoma estadío A, fueron sometidos a una LRLA-L.

Los datos fueron recolectados en forma prospectiva. Las variables analizadas se presentan en la Tabla 1.

En todos los casos se realizó un abordaje laparoscópico transperitoneal con 4 trocares, colocan-

Tabla 1. Variables analizadas

\begin{tabular}{l} 
Edad \\
Lado de la disección \\
Tiempo operatorio \\
Sangrado intraoperatorio \\
Tiempo de hospitalización \\
Complicaciones \\
Número de nodos linfáticos resecados \\
\hline
\end{tabular}

do al paciente en posición de lumbotomía de acuerdo al lado afectado (Figuras 1 y 2).

Para la disección se utilizaron los límites clásicos para la linfadenectomía con preservación de nervios (Figura 3).

\section{Resultados}

En el período analizado se obtuvieron un total de 118 pacientes, con una edad promedio de 29 años (rango 15-44). En 64 casos la disección fue del lado derecho mientras que en 54 casos esta fue izquierda. El tiempo operatorio promedio fue de 120

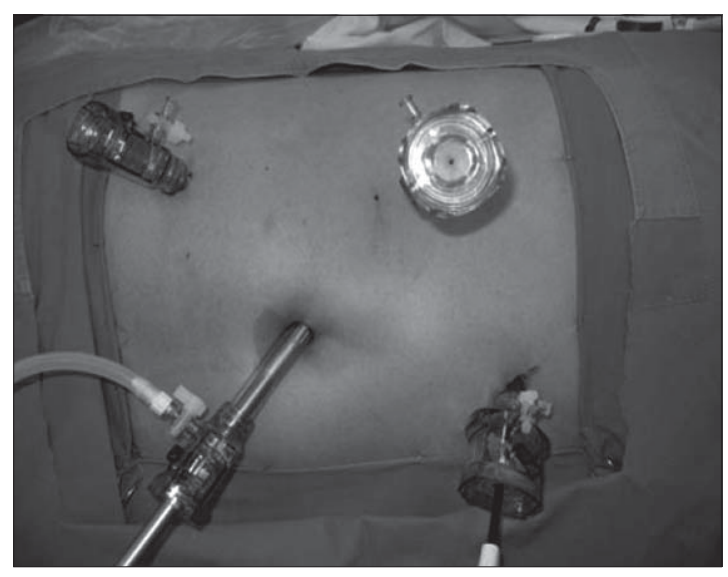

Figura 1. Posición de trocares lado derecho.

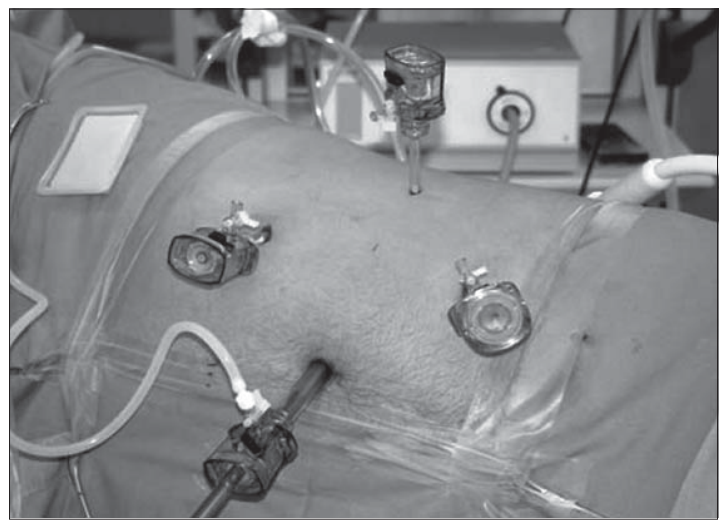

Figura 2. Posición de trocares lado izquierdo. 
minutos (rango 60-300), con un sangrado promedio de $50 \mathrm{cc}$ (rango 10 -1.000). El tiempo promedio de hospitalización fue de 41.4 horas (rango 12-120), y el promedio de nodos linfáticos resecados fue de 15 (rango 4-36) (Tabla 2).

Se presentaron complicaciones en 12 casos $(10,8 \%)$. El sangrado fue la complicación más frecuente, presentándose en 10 casos (8,5\%). Las lesiones vasculares se presentaron de la siguiente forma: 3 lesiones de vena cava, 3 lesiones de vena

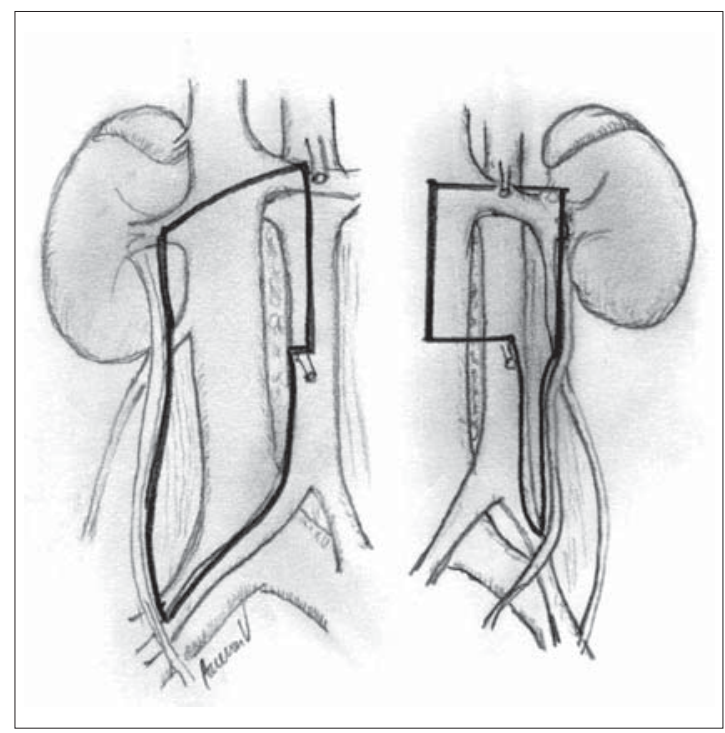

Figura 3. Límites de disección con preservación de nervios.

Tabla 2. Resultados

\begin{tabular}{ll}
$\begin{array}{l}\text { Número de casos } \\
\text { Edad }\end{array}$ & $\begin{array}{l}118 \\
29 \text { años } \\
\text { (rango de } 15 \text { a 44) }\end{array}$ \\
$\begin{array}{l}\text { Disección } \\
\text { Derecha } \\
\text { Izquierda } \\
\text { Sangrado promedio }\end{array}$ & $\begin{array}{l}64 \text { casos } \\
54 \text { casos }\end{array}$ \\
$\begin{array}{l}\text { Tiempo quirúrgico promedio } \\
\text { (rango } 10 \text { a 1.000) }\end{array}$ & $\begin{array}{l}120 \text { min } \\
\text { (rango } 60 \text { a 300) }\end{array}$ \\
$\begin{array}{l}\text { Tiempo de hospitalización } \\
\text { promedio }\end{array}$ & 41,4 horas \\
Número de nodos linfáticos & 15 nodos \\
resecados & (rango 4 a 36) \\
\hline
\end{tabular}

lumbar, 1 lesión de vena espermática, 1 lesión de arteria ilíaca, 1 lesión de arteria espermática y un hematoma retroperitoneal.

En un caso se produjo una lesión puntiforme de duodeno que se suturó por vía laparoscópica $(0,85 \%)$, mientras que otro paciente evolucionó con un edema pulmonar agudo $(0,85 \%)$.

De las 11 lesiones quirúrgicas, 8 fueron manejadas por vía laparoscópica (Tabla 3), mientras que en 3 casos $(2,5 \%)$, fue necesaria la conversión a cirugía abierta. Estos casos fueron: una lesión de vena cava por punción con el primer trocar, una lesión por quemadura de la arteria ilíaca y un desgarro de una vena lumbar. No hubo mortalidad en esta serie.

\section{Discusión}

La linfadenectomía retroperitoneal lumboaórtica continúa siendo el método más confiable para la detección de metástasis linfáticas en los pacientes portadores de un tumor de células germinales no seminoma etapa $\mathrm{A}^{1}$. Sin embargo, y como hemos mencionado previamente, la morbilidad asociada a este procedimiento es un aspecto importante a considerar. Es en este sentido que el abordaje laparoscópico nos ofrece una alternativa menos invasiva

Tabla 3. Complicaciones y manejo

\begin{tabular}{|c|c|}
\hline Complicación & Manejo \\
\hline Lesión de vena cava & Reparación laparoscópica \\
\hline Lesión de vena cava & Reparación laparoscópica \\
\hline Lesión de vena cava & $\begin{array}{l}\text { Reparación por cirugía } \\
\text { abierta }\end{array}$ \\
\hline Lesión de vena lumbar & Reparación laparoscópica \\
\hline Lesión de vena lumbar & Reparación laparoscópica \\
\hline Lesión de vena lumbar & $\begin{array}{l}\text { Reparación por cirugía } \\
\text { abierta }\end{array}$ \\
\hline $\begin{array}{l}\text { Lesión de vena } \\
\text { espermática }\end{array}$ & Reparación laparoscópica \\
\hline $\begin{array}{l}\text { Lesión de arteria } \\
\text { espermática }\end{array}$ & Reparación laparoscópica \\
\hline Lesión de arteria ilíaca & $\begin{array}{l}\text { Reparación por cirugía } \\
\text { abierta }\end{array}$ \\
\hline $\begin{array}{l}\text { Hematoma } \\
\text { retroperitoneal }\end{array}$ & Observación \\
\hline Lesión de duodeno & Sutura laparoscópica \\
\hline Edema pulmonar agudo & Manejo médico \\
\hline
\end{tabular}


Tabla 4. Comparación de parámetros operatorios

\begin{tabular}{|c|c|c|c|c|c|c|}
\hline & $\begin{array}{c}\text { Casos } \\
\text { (n) }\end{array}$ & $\begin{array}{c}\text { Tiempo } \\
\text { operatorio } \\
\text { (min) }\end{array}$ & $\begin{array}{c}n \\
\text { conversiones }\end{array}$ & $\begin{array}{l}\text { Sangrado } \\
(\mathrm{ml})\end{array}$ & $\begin{array}{l}\text { Complicaciones } \\
\text { intraoperatorias } \\
\text { mayores (n) }\end{array}$ & $\begin{array}{c}\text { Estadía } \\
\text { hospitalaria } \\
\text { (días) }\end{array}$ \\
\hline Albqami et al. ${ }^{4}$ & 103 & 217 & 3 & 144 & 3 & 3,6 \\
\hline Rassweiler et al. ${ }^{5}$ & 34 & 249 & 1 & - & 1 & 5,3 \\
\hline Porter et al. ${ }^{9}$ & 27 & 306 & 0 & 270 & - & 2,1 \\
\hline LeBlanc et al. ${ }^{6}$ & 20 & 230 & 0 & $<50$ & 0 & 1,2 \\
\hline Corvin et al. ${ }^{7}$ & 18 & 232 & 0 & $<100$ & 0 & - \\
\hline Castillo et al. & 118 & 120 & 3 & 50 & 11 & 1,8 \\
\hline
\end{tabular}

y comparable, desde el punto de vista de la morbilidad, con la cirugía abierta ${ }^{1}$. Sin bien es cierto, la morbilidad asociada a la LRLA ha disminuido en forma considerable, las series de pacientes sometidos a LRLA-L muestran menor morbilidad postoperatoria, menos sangrado intraoperatorio, menor estadía hospitalaria y convalecencia más rápida ${ }^{2,3}$. Janetscheck y cols ${ }^{4}$, en su serie de 103 pacientes reportan un tiempo operatorio promedio de $217 \mathrm{mi}$ nutos con un sangrado promedio de $144 \mathrm{ml}$ y una estadía hospitalaria promedio de 3,6 días. Si analizamos las series publicadas donde se reportan $10 \mathrm{o}$ más casos, con un seguimiento de al menos 24 me$\operatorname{ses}^{4-9}$, los parámetros operatorios varían de la siguiente forma; tiempo operatorio entre 138 y 306 minutos, con un $3,1 \%$ de conversión a cirugía abierta; sangrado intraoperatorio en un rango entre 50 $\mathrm{ml}$ y $390 \mathrm{ml}$, con un $1 \%$ de pacientes que requieren transfusión sanguínea, y finalmente una estadía hospitalaria que varía entre 1,8 a 5,3 días.

Estos resultados se comparan de forma favorable con los de la serie más grande de LRLA, con un total de 239 pacientes, presentando un tiempo operatorio promedio de 214 minutos con un sangrado promedio de $150 \mathrm{ml}$ y una estadía hospitalaria promedio de 8 días $^{10}$.

En la LRLA-L las complicaciones intraoperatorias mayores y menores se presentan en un $4 \%$ y un $2 \%$ respectivamente, mientras que en la LRLA, las complicaciones mayores y menores se ven en un $5 \%$ y un $15 \%$ de los casos. Las complicaciones más frecuentes son las lesiones vasculares, siendo también las responsables de la conversión a cirugía abierta en la mayoría de los casos ${ }^{1}$. Romero y cols $^{11}$, presentan una serie de 77 pacientes sometidos a LRLA-L, 10 (13\%) de los cuales presentaron complicaciones intraoperatorias. Las lesiones de vena cava $(5,4 \%)$, hilio renal $(3,1 \%)$, y arteria ilíaca externa (1\%) fueron las más frecuentes.

De igual forma los resultados de nuestra serie, tanto en parámetros operatorios como en morbilidad, son comparables a los de las series antes mencionadas, tanto de LRLA como de LRLA-L (Tabla 4).

En síntesis, podemos decir, que la linfadenectomía lumboaórtica laparoscópica es un procedimiento estandarizado, que en manos experimentadas presenta una tasa de complicaciones aceptable, siendo la más frecuente el sangrado.

Además, en base a nuestra experiencia y tomando en cuenta los bajos porcentajes de conversión a cirugía abierta, nos parece que la mayoría de estas complicaciones puede ser resuelta en forma laparoscópica.

Finalmente, debemos tomar en cuenta que esta serie prospectiva considera la curva de aprendizaje autodidacta y el entrenamiento de urólogos en formación en laparoscopía.

\section{Referencias}

1. Hamilton R, Finelli A. Laparoscopic Retroperitoneal Lymph Node Dissection for Nonseminomatous Germ Cell Tumors: Current Status. Urol Clin N Am 2007; 34: 159-169.

2. Giusti G, Beltrami P, Tallarigo C. Unilateral laparoscopic retroperitoneal limphadenectomy for clinical stage I nonseminomatous testicular cancer. J Endourol 1998; 12: 561-566.

3. Carver BS, Scheinfeld J. The current status of laparoscopic retroperitoneal limph node dissection for nonseminomatous germ-cell tumors. Nat Clin Pract Urol 2005; 2: 330-335.

4. Albqami N, Janetschek G. Laparoscopic retroperitoneal limph-node dissection in the management of clinical stage I and II testicular cancer. J Endourol 2005; 19: 683-692.

5. Rassweiler JJ, Frede T, Lenz E. Long-term experience with laparoscopic retroperitoneal lymph node dissection 
in the management of low stage testis cancer. Eur Urol 2000; 37: 251-260.

6. LeBlanc E, Cathy A, Dargent D. Extraperitoneal laparoscopic para-aortic lymph node dissection for early stage nonseminomatous germ cell tumors of the testis with introduction of a nerve sparing technique description and results. J Urol 2001; 165: 89-92.

7. Corvin S, Sturm W, Schlatter E. Laparoscopic retroperitoneal lymph-node dissection with the waterjet is technically feasible and safe in testis cancer patient. J Endourol 2005; 19: 823-826.

8. Castillo OA, Urena RD, Pinto IF. Laparoscopic retroperitoneal lymph node dissection for stage I and II NSGCT: 10 year's experience. J Urol 2004; 171
(Suppl): 247-248.

9. Porter JR, Lange PH. Laparoscopic retroperitoneal lymph node dissection for stage I NSGCT (abstract \# 302). Presented at the American Urological Association National Meeting Chicago, IL; April 27, 2003.

10. Heidenreich A, Albers P, Hartmann M. Complications of primary nerve sparing retroperitoneal lymph node dissection for clinical stage I nonseminomatous germ cell tumors of the testis: experience of the German Testicular Cancer Study Group. J Urol 2003; 169: 17101714.

11. Romero FR, Wagner A, Brito FA. Refining the laparoscopic retroperitoneal lymph node dissection for testicular cancer. Int Braz J Urol 2006; 32: 196-201. 